\section{SDC4: OA joint effort}

By Michael J. Haas, Senior Writer

Most approved osteoarthritis therapies are NSAIDs that treat the symptoms but do not halt the progress of the disease. Indeed, joint replacement surgery is the only curative strategy. Now, a team of German and South Korean researchers has shown that inhibiting syndecan-4 prevents cartilage damage in mice. ${ }^{1}$ The findings point to a strategy for halting - if not reversing-disease progression.

Disease modifiers would be especially welcome in OA because the disease is difficult to detect until damage has occurred. The search for targets involved in the pathogenesis of OA thus far has yielded little fruit, in part because the disease has multiple genetic components that are not well understood.

Aggrecanases, such as the ADAM family of metallopeptidases, emerged as promising targets in 2005, when separate teams from Wyeth and from The University of Melbourne and The Royal Children's Hospital (RCH) reported in Nature that mouse models of OA deficient in ADAMTS5 had less cartilage damage than wild-type controls. ${ }^{2,3}$

However, ADAMTS5 (ADAM metallopeptidase with thrombospondin type 1 motif 5) and other metallopeptidases are involved in many normal biological processes, making them difficult to target selectively to treat disease.

To find more selective targets, researchers from Germany and South Korea looked for ADAM enzyme activators that were expressed in cartilage cells. Syndecans, a family of four heparan sulfate proteoglycans, fit the bill because they were already known to activate several ADAM enzymes.

In particular, the group focused on syndecan-4 (SDC4), which is known to regulate mesenchymal stem cells (MSCs) during tissue repair and to interact with other extracellular matrix proteins.

Indeed, the team found that SDC4 was upregulated in cartilage samples from the joints of OA patients and in two mouse models of OA compared with what was seen in controls. Blocking the target protected mice from articular (joint) cartilage degradation compared with that in control models. Cartilage samples from the Sdc4-deficient models also exhibited fewer signs of aggrecanase activity, suggesting that Sdc4 played a role in activating those enzymes.

Studies in mouse cartilage samples confirmed that Sdc4 inhibition downregulated the activity of matrix metalloproteinase 3 (Mmp3), which in turn prevented Adamts5 activation and cartilage destruction.

Collectively the results indicate that SDC4 plays a cell- and disease-specific role in $\mathrm{OA}$, thus making it a more feasible target than
ADAMTS5 or MMP3, team coleader Thomas Pap told SciBX. Pap is professor of experimental medicine and director of the Institute of Experimental Musculoskeletal Medicine at University Hospital Munster.

The team was also led by Gregor Theilmeier, professor of anesthesiology and director of research in anesthesiology at Hannover Medical School. Other authors on the paper, which appeared in Nature Medicine, were from University Medical Center Hamburg-Eppendorf and Seoul National University College of Medicine.

\section{Bones to pick}

Pap said upcoming publications will report the results of the team's ongoing studies of SDC4's role in joint remodeling. The group is also investigating whether SDC4 inhibition can help regenerate damaged cartilage in $\mathrm{OA}$ and thus reverse the course of disease.

"Interfering with the SDC4 signaling pathway could be a promising strategy for delaying cartilage damage in $\mathrm{OA}$ and could represent a valuable approach to treat OA in combination with other therapies," said Manlio Bolla, director of scientific affairs at NicOx S.A.

NicOx's naproxcinod (HCT 3012), a cyclooxygenase (COX)-inhibiting nitric oxide donator (CINOD) that releases naproxen and nitric oxide, has completed three Phase III trials to treat OA. The company expects to submit an NDA to the FDA and an MAA to the EMEA in the third and fourth quarters, respectively.

"Regulation of cartilage breakdown is regarded as the most important challenge in developing therapies for osteoarthritis," and the authors of the Nature Medicine paper showed that SDC4 could be a valid, druggable target to achieve that, said Seonggu Ro, EVP and CTO of CrystalGenomics Inc. "However, because SDC4's mechanism of action is not related to the control of inflammation or pain, patients who would take SDC4 blockers might also need NSAID treatment."

CrystalGenomics' CG100649, a dual inhibitor of COX-2 and carbonic anhydrase I (CAI), has completed Phase IIa testing to treat OA.

Bolla wanted to see safety studies that examine the effects of SDC4 inhibition in other systems and tissues because SDC4 is not specific to cartilage. "Syndecans have been implicated in all sorts of cell-cell interactions, including migration and metastatic processes in cancer, and are involved in cytoskeletal organization," he noted.

Bolla added that the Sdc4 knockout mice "have shown defects in wound repair, in which SDC4 seems to be implicated."

Pap acknowledged that more work is needed to assess the safety of systemic SDC4 inhibition, as the mice in his team's study received the anti-Sdc4 antibody by intra-articular injection and only for a few weeks.

Nevertheless, he thinks that SDC4 inhibitors are likely to have a good safety profile. "Sdc4 knockout mice are viable with no overt phenotype" apart from a few days' delay in wound healing due to slower differentiation of myofibroblasts, ${ }^{4}$ he said. "But that effect is very mild, which suggests systemic inhibition of SDC4 should not have severe side effects." 


\section{COVER STORY}

The findings reported in Nature Medicine are not yet patented but are available for licensing, Pap told SciBX.

Haas, M.J. SciBX 2(34); doi:10.1038/scibx.2009.1297

Published online Sept. 3, 2009

\section{REFERENCES}

1. Echtermeyer, F. Nat. Med.; published online Aug. 16, 2009; doi:10.1038/nm.1998

Contact: Frank Echtermeyer, Hannover Medical School, Hannover, Germany

e-mail: echtermeyer.frank@mh-hannover.de

Contact: Thomas Pap, University Hospital Munster, Munster, Germany e-mail: Thomas.pap@uni-muenster.de
2. Glasson, S. et al. Nature 434, 644-648 (2005)

3. Stanton, H. et al. Nature 434, 648-652 (2005)

4. Echtermeyer, F. et al. J. Clin. Invest. 107, R9-R14 (2001)

COMPANIES AND INSTITUTIONS MENTIONED CrystalGenomics Inc. (KOSDAQ:083790), Seoul, South Korea Hannover Medical School, Hannover, Germany NicOx S.A. (Euronext:COX), Sophia-Antipolis, France

The Royal Children's Hospital, Melbourne, Victoria, Australia University Hospital Munster, Munster, Germany

University Medical Center Hamburg-Eppendorf, Hamburg, Germany The University of Melbourne, Melbourne, Victoria, Australia Seoul National University College of Medicine, Seoul, South Korea Wyeth (NYSE:WYE), Madison, N.J. 